Sparkes, H. A., Forrest, S. J. K., \& Lorenzo, D. A. (2016). Crystal engineering: co-crystals of cinnamic acid derivatives with a pyridyl derivative co-crystalliser. Acta Crystallographica Section B: Structural Science, Crystal Engineering and Materials, 72(1), 87-95. https://doi.org/10.1107/S2052520615019678, https://doi.org/10.1107/S2052520615019678/bm5077sup1.cif, https://doi.org/10.1107/S2052520615019678/bm50771Asup2.hkl, https://doi.org/10.1107/S2052520615019678/bm50771Bsup3.hkl, https://doi.org/10.1107/S2052520615019678/bm50771Csup4.hkl, https://doi.org/10.1107/S2052520615019678/bm50772Asup5.hkl, https://doi.org/10.1107/S2052520615019678/bm50772Bsup6.hkl, https://doi.org/10.1107/S2052520615019678/bm50773Asup7.hkl, https://doi.org/10.1107/S2052520615019678/bm50773Bsup8.hkl, https://doi.org/10.1107/S2052520615019678/bm50774Bsup9.hkl, https://doi.org/10.1107/S2052520615019678/bm5077sup10.pdf

Publisher's PDF, also known as Version of record

Link to published version (if available):

$10.1107 / S 2052520615019678$

10.1107/S2052520615019678/bm5077sup1.cif

10.1107/S2052520615019678/bm50771Asup2.hkl 10.1107/S2052520615019678/bm50771Bsup3.hkl 10.1107/S2052520615019678/bm50771Csup4.hkl 10.1107/S2052520615019678/bm50772Asup5.hkl 10.1107/S2052520615019678/bm50772Bsup6.hkl 10.1107/S2052520615019678/bm50773Asup7.hkl 10.1107/S2052520615019678/bm50773Bsup8.hkl 10.1107/S2052520615019678/bm50774Bsup9.hkl 10.1107/S2052520615019678/bm5077sup10.pdf

Link to publication record in Explore Bristol Research

PDF-document

This is the final published version of the article (version of record). It first appeared online via International Union of Crystallography at http://journals.iucr.org/b/issues/2016/01/00/bm5077/index.html. Please refer to any applicable terms of use of the publisher. 
STRUCTURAL SCIENCE CRYSTAL ENGINEERING MATERIALS

ISSN 2052-5206

Received 14 August 2015

Accepted 17 October 2015

Edited by A. J. Blake, University of Nottingham, England

Keywords: co-crystal; cinnamic acid; solvent drop grinding.

CCDC references: $1415119 ; 1415120$; $1415121 ; 1415122 ; 1415123 ; 1415124$; $1415125 ; 1415126$

Supporting information: this article has supporting information at journals.iucr.org/b

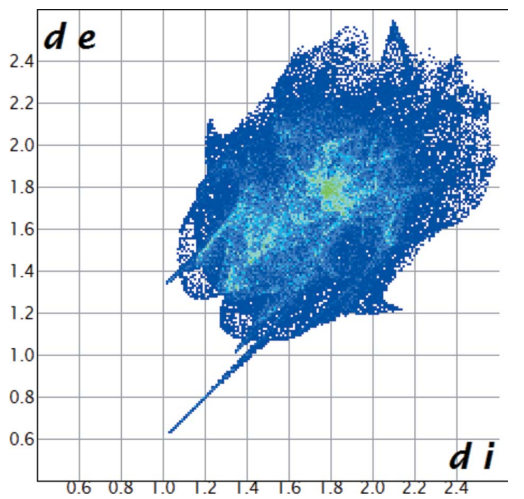

C 2016 International Union of Crystallography

\section{Crystal engineering: co-crystals of cinnamic acid derivatives with a pyridyl derivative co-crystallizer}

\author{
Daniel A. Lorenzo, Sebastian J. K. Forrest and Hazel A. Sparkes*
}

Department of Chemistry, University of Bristol, Cantock's Close, Bristol BS8 1TS, England. *Correspondence e-mail: hazel.sparkes@bristol.ac.uk

A number of hydrogen-bonded co-crystals, consisting of a cinnamic acid derivative and a pyridyl co-crystallizer, have been synthesized and their properties investigated by X-ray diffraction. Samples were prepared by recrystallization or solvent drop grinding of trans-cinnamic acid (1), 4methylcinnamic acid (2), 4-methoxy cinnamic acid (3) or 3,4-methoxy cinnamic acid (4), with 4,4-dipyridyl $(A)$, iso-nicotinamide $(B)$ or nicotinamide $(C)$. The $\mathrm{X}$-ray single-crystal structures of seven novel co-crystals, obtained through recrystallization, are examined and the hydrogen-bonding interactions discussed. Consistent hydrogen-bonding motifs were observed for samples prepared when using 4,4-dipyridyl $(A)$ or iso-nicotinamide $(B)$ as the cocrystallizing agent. Powder X-ray diffraction analysis of the samples prepared by solvent drop grinding suggests the formation of ten co-crystals.

\section{Introduction}

The formation of co-crystals is of great importance in a wide range of fields, for example optoelectronics (Zhu et al., 2014, 2015), pharmaceuticals (Brittain, 2012; Steed, 2013) and gas storage or separation (Jones et al., 2011), allowing the properties of compounds and structures to be tuned. While much discussion has gone into the precise nature of co-crystals (Aakeröy et al., 2007; Aitipamula et al., 2012), a widely accepted description is that co-crystals consist of two or more neutral components held together by non-covalent interactions, e.g. hydrogen bonding. Given that no covalent bonds are formed or broken in the preparation of a co-crystal, the synthetic design process requires an understanding of intermolecular interactions (Allen et al., 1998, 1999), in order to use crystal engineering (Aakeröy, 1997) approaches based on supramolecular synthons (Hemamalini et al., 2014; MoraguesBartolome et al., 2012; Bučar et al., 2014; Aakeröy et al., 2012). Alongside the preparation of co-crystals through recrystallization, which is limited by the solubility of the components in different solvents (Braga et al., 2013; James et al., 2012), another method for co-crystal preparation is through mechanochemical grinding of the two species (Braga et al., 2013). The advantage of such an approach is that it is generally environmentally friendly, avoiding the use of large amounts of solvent and usually resulting in a singular product (James et al., 2012).

Co-crystals can possess different physical properties to their respective individual components, for example their habit, solubility, melting point and dissolution rate (Brittain, 2012; Elder et al., 2013). Within the pharmaceutical industry a common problem associated with bringing API's (active pharmaceutical ingredients) to market is poor bioavailability. Pharmaceutical co-crystals which consists of at least one 
component that is an API can result in a modification of the physiochemical properties, e.g. solubility of the API, and thus affect the bioavailability of the species without altering the API's activity (Steed, 2013). Clearly the co-former in the cocrystal needs to be safe for human consumption with no side effects.

The formation of co-crystals between cinnamic acid derivatives and iso-nicotinamide has previously been carried out resulting in the formation of 12 co-crystals (Aakeröy et al., 2002). Two distinctive supramolecular hydrogen-bonding motifs were identified in the co-crystals, the first involving the carboxylic acid $\mathrm{OH}$ and the $\mathrm{N}$ of the pyridyl, and the second amide-amide hydrogen-bonding interactions. In addition one hydrate of 4-hydroxy-3-methoxycinnamic acid and isonicotinamide has been reported (Clarke et al., 2010); however, this contained amide-carboxylic acid $\mathrm{OH} \cdots \mathrm{O}$ and $\mathrm{NH} \cdots \mathrm{O}$ hydrogen bonding rather than amide-amide and carboxylic acid-dipyridyl hydrogen bonding.

Two polymorphic co-crystals of 4-hydroxycinnamic acid ( $p$ coumaric acid) and nicotinamide have previously been reported (Bevill et al., 2014). Both of these contain $\mathrm{OH} \cdots \mathrm{O}$ and $\mathrm{NH} \cdots \mathrm{O}$ hydrogen bonding between the carboxylic acid and the amide $\mathrm{CONH}_{2}$, with the hydroxyl $\mathrm{OH}$ group forming a hydrogen-bonding interaction to the $\mathrm{N}$ of the pyridyl group. Two further hydrates of co-crystals containing nicotinamide and a cinnamic acid derivative (3,4-dihydroxy cinnamic acid and 4-hydroxy-3-methoxycinnamic acid; Clarke et al., 2010) also contained $\mathrm{OH} \cdots \mathrm{O}$ and $\mathrm{NH} \cdots \mathrm{O}$ hydrogen bonding rather than amide-amide and carboxylic acid-dipyridyl hydrogen bonding.

As part of a wider program of research into the crystalline behaviour of cinnamic acid derivatives (Allen et al., 2005; Howard et al., 2009; Mahon et al., 2008; Yates \& Sparkes, 2013), the interaction of four cinnamic acid derivatives [(1)(4)] with three potential co-crystallizers $(A)-(C)$ has been investigated in order to obtain further insight into the supramolecular structural motifs formed. Co-crystallizers were selected on their potential to form hydrogen-bonding interactions with the carboxylic acid group (Aakeröy et al., 2002; Seaton, 2011) of the selected cinnamic acids and thus form a co-crystal, see Fig. 1. The results of the preparation of the series of cinnamic acid crystals via recrystallization and solvent drop grinding are reported.

\section{Experimental}

trans-Cinnamic acid (1), 4-methylcinnamic acid (2), 4-methoxycinnamic acid (3), 3,4-methoxycinnamic acid (4), 4,4dipyridyl $(A)$, iso-nicotinamide $(B)$ and nicotinamide $(C)$ along with all solvents were used as received without further purification from Sigma Aldrich.

\subsection{General procedure for recrystallization}

Small amounts of a cinnamic acid derivative (1)-(4) and a co-crystallizer $(A)-(C)$ in the appropriate stoichiometric ratio (i.e. 2:1 for cinnamic acid:dipyridyl and 1:1 for cinnamic acid:iso-nicotinamide and cinnamic acid:nicotinamide) were dissolved in a solvent in which both species were soluble. The vials were left for the solvent to evaporate slowly over a couple of weeks to yield crystals which were analysed by single-crystal X-ray diffraction.

2.2. General procedure for sample preparation by ball milling and solvent drop grinding

Small stoichiometric amounts of a cinnamic acid derivative (1)-(4) and a co-crystallizer $(A)-(C)$ in the appropriate stoichiometric ratio (i.e. 2:1 for cinnamic acid:dipyridyl and 1:1 for cinnamic acid:iso-nicotinamide and cinnamic acid:nicotinamide) totalling $\sim 0.12 \mathrm{~g}$ were loaded into a Retsch MM200 ball mill with $\sim 0.04 \mathrm{ml}$ of ethanol. The samples were ground in the ball mill for $30 \mathrm{~min}$ at a frequency of $10 \mathrm{~Hz}$. The samples were then analysed using powder X-ray diffraction to check for signs of the formation of a co-crystal.

\subsection{Single-crystal X-ray diffraction}

Single-crystal X-ray diffraction data collections on $(1 A)$, $(1 B),(1 C),(2 A),(2 B),(3 A),(3 B)$ and $(4 B)$ were carried out at $100 \mathrm{~K}$ on a Bruker APEXII diffractometer using Mo $K \alpha$ radiation $(\lambda=0.71073 \AA)$. Data collections were performed using a CCD area detector from a single crystal mounted on a glass fibre. Absorption corrections were based on equivalent reflections using $S A D A B S$ (Bruker, 2001). All of the structures were solved using SUPERFLIP (Palatinus \& Chapuis, 2007; Palatinus et al., 2012; Palatinus \& van der Lee, 2008), and refined against $F^{2}$ in SHELXL (Sheldrick, 2015) using OLEX2 (Dolomanov et al., 2009). All of the $\mathrm{O}-\mathrm{H}$ or $\mathrm{N}-\mathrm{H}$ hydrogen atoms were located in the difference map and the majority refined freely; there were a couple of exceptions which required some restraints on the $D-\mathrm{H}$ distance and the $\mathrm{H}$ atoms attached to the disordered fragment in $(2 A)$ which were fixed geometrically and refined using a riding model; the

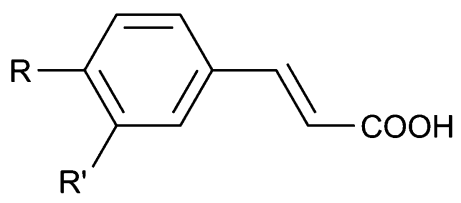

(1) $\mathrm{R}=\mathrm{H}, \mathrm{R}^{\prime}=\mathrm{H}$; (2) $\mathrm{R}=\mathrm{Me}, \mathrm{R}^{\prime}=\mathrm{H}$; (3) R = OMe, R' = H; (4) $\mathrm{R}=\mathrm{OMe}, \mathrm{R}^{\prime}=\mathrm{OMe}$

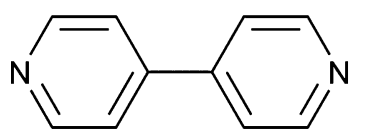

A

(A)

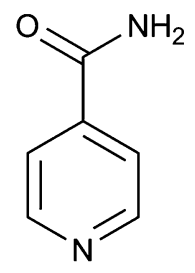

(B)

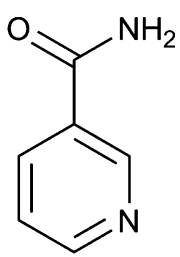

(C)
Figure 1

Four cinnamic acid derivatives, trans-cinnamic acid (1), 4-methylcinnamic acid (2), 4-methoxy cinnamic acid (3) and 3,4-methoxy cinnamic acid (4). Three co-crystallizers: 4,4-dipyridyl $(A)$, iso-nicotinamide $(B)$ and nicotinamide $(C)$. 
Table 1

Experimental details.

\begin{tabular}{|c|c|c|c|c|c|}
\hline & $(1 A)$ & $(1 B)$ & $(1 C)$ & $(2 A)$ & $(2 B)$ \\
\hline \multicolumn{6}{|l|}{ Crystal data } \\
\hline Chemical formula & $\mathrm{C}_{10} \mathrm{H}_{8} \mathrm{~N}_{2} \cdot 2 \mathrm{C}_{9} \mathrm{H}_{8} \mathrm{O}_{2}$ & $\mathrm{C}_{9} \mathrm{H}_{8} \mathrm{O}_{2} \cdot \mathrm{C}_{6} \mathrm{H}_{6} \mathrm{~N}_{2} \mathrm{O}$ & $\mathrm{C}_{9} \mathrm{H}_{8} \mathrm{O}_{2} \cdot \mathrm{C}_{6} \mathrm{H}_{6} \mathrm{~N}_{2} \mathrm{O}$ & $2 \mathrm{C}_{10} \mathrm{H}_{10} \mathrm{O}_{2} \cdot \mathrm{C}_{10} \mathrm{H}_{8} \mathrm{~N}_{2}$ & $\mathrm{C}_{10} \mathrm{H}_{10} \mathrm{O}_{2} \cdot \mathrm{C}_{6} \mathrm{H}_{6} \mathrm{~N}_{2} \mathrm{O}$ \\
\hline$M_{\mathrm{r}}$ & 452.49 & 270.28 & 270.28 & 480.54 & 284.31 \\
\hline $\begin{array}{l}\text { Crystal system, space } \\
\text { group }\end{array}$ & Monoclinic, $P 2_{1} / n$ & Triclinic, $P \overline{1}$ & Monoclinic, $P 2_{1} / c$ & Triclinic, $P \overline{1}$ & Triclinic, $P \overline{1}$ \\
\hline Temperature (K) & 100 & 100 & 100 & 100 & 100 \\
\hline$a, b, c(\AA)$ & $\begin{array}{l}7.7952(2), 7.1669(2), \\
20.7708(5)\end{array}$ & $\begin{array}{l}8.4176(3), 10.6487(4) \\
15.0679(5)\end{array}$ & $\begin{array}{l}14.0191(7), 6.1871(3) \\
16.6387(7)\end{array}$ & $\begin{array}{l}10.8843(2), 15.6510(4), \\
16.6073(4)\end{array}$ & $\begin{array}{l}7.0311(2), 8.6172(2), \\
11.9490(3)\end{array}$ \\
\hline$\alpha, \beta, \gamma\left({ }^{\circ}\right)$ & $90,93.2075(17), 90$ & $\begin{array}{l}75.883(2), 82.329(2) \\
\quad 83.301(2)\end{array}$ & $90,111.740(3), 90$ & $\begin{array}{l}63.358(1), 79.726(1) \\
\quad 77.722(1)\end{array}$ & $\begin{array}{l}77.4101(14) \\
87.9565(14) \\
82.6358(14)\end{array}$ \\
\hline$V\left(\AA^{3}\right)$ & $1158.59(5)$ & $1293.14(8)$ & $1340.56(11)$ & $2459.66(10)$ & $700.71(3)$ \\
\hline$Z$ & 2 & 4 & 4 & 4 & 2 \\
\hline$F(000)$ & 476 & 568 & 568 & 1016 & 300 \\
\hline Radiation type & Mo $K \alpha$ & Mo $K \alpha$ & Mo $K \alpha$ & Mo $K \alpha$ & Mo $K \alpha$ \\
\hline$D_{x}\left(\mathrm{Mg} \mathrm{m}^{-3}\right)$ & 1.297 & 1.388 & 1.339 & 1.298 & 1.347 \\
\hline$\mu\left(\mathrm{mm}^{-1}\right)$ & 0.09 & 0.10 & 0.10 & 0.09 & 0.09 \\
\hline Crystal size (mm) & $0.63 \times 0.32 \times 0.3$ & $0.6 \times 0.47 \times 0.33$ & $0.22 \times 0.15 \times 0.13$ & $0.67 \times 0.4 \times 0.27$ & $0.62 \times 0.35 \times 0.28$ \\
\hline \multicolumn{6}{|l|}{ Data collection } \\
\hline Diffractometer & $\begin{array}{l}\text { Bruker APEXII kappa } \\
\text { CCD area detector }\end{array}$ & $\begin{array}{l}\text { Bruker APEXII kappa } \\
\text { CCDC area detector }\end{array}$ & $\begin{array}{l}\text { Bruker APEXII kappa } \\
\text { CCD area detector }\end{array}$ & $\begin{array}{l}\text { Bruker APEXII kappa } \\
\text { CCD area detector }\end{array}$ & $\begin{array}{c}\text { Bruker APEXII kappa } \\
\text { CCD area detector }\end{array}$ \\
\hline Absorption correction & $\begin{array}{l}\text { Multi-scan } S A D A B S \\
\quad(\text { Bruker, 2001) }\end{array}$ & $\begin{array}{l}\text { Multi-scan } S A D A B S \\
\quad \text { (Bruker, 2001) }\end{array}$ & $\begin{array}{l}\text { Multi-scan } S A D A B S \\
\quad \text { (Bruker, 2001) }\end{array}$ & $\begin{array}{l}\text { Multi-scan } S A D A B S \\
\quad \text { (Bruker, 2001) }\end{array}$ & $\begin{array}{l}\text { Multi-scan } S A D A B S \\
\quad \text { (Bruker, 2001) }\end{array}$ \\
\hline$T_{\min }, T_{\max }$ & $0.658,0.746$ & $0.624,0.746$ & $0.645,0.746$ & $0.593,0.746$ & $0.664,0.746$ \\
\hline $\begin{array}{l}\text { No. of measured, inde- } \\
\text { pendent and observed } \\
{[I>2 \sigma(I)] \text { reflections }}\end{array}$ & $10075,2759,2356$ & $11995,6048,4955$ & $11465,3188,2166$ & $45365,11862,8260$ & $12831,3359,3003$ \\
\hline$R_{\text {int }}, R_{\text {sigma }}$ & $0.022,0.021$ & $0.017,0.026$ & $0.054,0.051$ & $0.045,0.044$ & $0.016,0.014$ \\
\hline$(\sin \theta / \lambda)_{\max }\left(\AA^{-1}\right)$ & 0.658 & 0.660 & 0.658 & 0.660 & 0.658 \\
\hline \multicolumn{6}{|l|}{ Refinement } \\
\hline $\begin{array}{l}R\left[F^{2}>2 \sigma\left(F^{2}\right)\right], w R\left(F^{2}\right) \\
\quad\end{array}$ & $0.036,0.095,1.06$ & $0.039,0.110,1.03$ & $0.045,0.108,1.00$ & $0.051,0.138,1.02$ & $0.035,0.101,1.04$ \\
\hline No. of reflections & 2759 & 6048 & 3188 & 11862 & 3359 \\
\hline No. of parameters & 158 & 385 & 193 & 740 & 203 \\
\hline No. of restraints & 0 & 0 & 0 & 331 & 0 \\
\hline H-atom treatment & $\begin{array}{l}\text { H atoms treated by a } \\
\text { mixture of indepen- } \\
\text { dent and constrained } \\
\text { refinement }\end{array}$ & $\begin{array}{l}\text { H atoms treated by a } \\
\text { mixture of indepen- } \\
\text { dent and constrained } \\
\text { refinement }\end{array}$ & $\begin{array}{l}\text { H atoms treated by a } \\
\text { mixture of indepen- } \\
\text { dent and constrained } \\
\text { refinement }\end{array}$ & $\begin{array}{l}\text { H atoms treated by a } \\
\text { mixture of indepen- } \\
\text { dent and constrained } \\
\text { refinement }\end{array}$ & $\begin{array}{l}\mathrm{H} \text { atoms treated by a } \\
\text { mixture of indepen- } \\
\text { dent and constrained } \\
\text { refinement }\end{array}$ \\
\hline$\Delta \rho_{\max }, \Delta \rho_{\min }\left(\mathrm{e} \AA^{-3}\right)$ & $0.38,-0.20$ & $0.46,-0.24$ & $0.23,-0.22$ & $0.81,-0.35$ & $0.44,-0.19$ \\
\hline
\end{tabular}

$$
(3 A)
$$

$(3 B)$

$(4 B)$

\section{Crystal data}

Chemical formula

$M_{\mathrm{r}}$

Crystal system, space group

Temperature $(\mathrm{K})$

$a, b, c(\AA)$

$\alpha, \beta, \gamma\left(^{\circ}\right)$

$V\left(\AA^{3}\right)$

$Z$

$F(000)$

$D_{x}\left(\mathrm{Mg} \mathrm{m}^{-3}\right)$

$\mu\left(\mathrm{mm}^{-1}\right)$

Crystal size (mm)

Data collection

Diffractometer

Absorption correction

$T_{\min }, T_{\max }$

No. of measured, independent and observed $[I>2 \sigma(I)]$ reflections

$R_{\text {int }}, R_{\text {sigma }}$

$(\sin \theta / \lambda)_{\max }\left(\AA^{-1}\right)$
$2 \mathrm{C}_{10} \mathrm{H}_{10} \mathrm{O}_{3} \cdot \mathrm{C}_{10} \mathrm{H}_{8} \mathrm{~N}_{2}$

512.54

Monoclinic, $P 2_{1} / n$

100

6.4681 (3), 28.9773 (13), 6.8930 (3)

90, $103.812(2), 90$

$1254.58(10)$

2

540

1.357

0.10

$0.4 \times 0.35 \times 0.35$

Bruker APEXII kappa CCD area detector

Multi-scan SADABS (Bruker, 2001)

$0.637,0.746$

$22702,3014,2677$

$0.032,0.019$

0.661
$\mathrm{C}_{10} \mathrm{H}_{10} \mathrm{O}_{3} \cdot \mathrm{C}_{6} \mathrm{H}_{6} \mathrm{~N}_{2} \mathrm{O}$

300.31

Triclinic, $P \overline{1}$

100

9.1225 (4), 11.5523 (4), 14.1938 (5)

90.700 (3), 90.577 (3), 104.055 (3)

$1450.81(10)$

4

632

1.375

0.10

$0.35 \times 0.2 \times 0.13$

Bruker APEXII kappa CCD area detector

Multi-scan $S A D A B S$ (Bruker, 2001)

$0.560,0.746$

$26528,6900,4151$

$0.070,0.068$

0.658

\section{$\mathrm{C}_{11} \mathrm{H}_{12} \mathrm{O}_{4} \cdot \mathrm{C}_{6} \mathrm{H}_{6} \mathrm{~N}_{2} \mathrm{O}$}

330.33

Monoclinic, $P 2_{1} / c$

100

14.0200 (8), 9.8795 (5), 11.6434 (6)

$90,96.975(4), 90$

$1600.80(15)$

4

696

1.371

0.10

$0.37 \times 0.27 \times 0.16$

Bruker APEXII kappa CCD area detector

Multi-scan SADABS (Bruker, 2001) $0.672,0.746$

$14321,3896,2262$

$0.085,0.086$

0.663 
Table 2 (continued)

$(3 A)$

$0.041,0.107,1.07$

3014

177

0

$\mathrm{H}$ atoms treated by a mixture of independent and constrained refinement

$0.35,-0.25$
$(3 B)$

$\begin{array}{ll}0.057,0.141,0.99 & 0.055,0.134,1.00 \\ 6900 & 3896 \\ 423 & 231 \\ 1 & 1 \\ \text { H atoms treated by a mixture of } & \text { H atoms treated by a mixture of } \\ \quad \text { independent and constrained } & \text { independent and constrained } \\ \quad \text { refinement } & \text { refinement } \\ 0.39,-0.32 & 0.25,-0.32\end{array}$

Table 2

Results of co-crystallization experiments.

\begin{tabular}{lllll}
\hline $\begin{array}{l}\text { Cinnamic } \\
\text { acid }\end{array}$ & Co-crystallizer & $\begin{array}{l}\text { Co-crystal } \\
\text { formed }\end{array}$ & Recrystallization & $\begin{array}{l}\text { Solvent } \\
\text { drop grinding }\end{array}$ \\
\hline$(1)$ & $(A)$ & $(1 A)$ & Co-crystal formed & Co-crystal formed \\
$(1)$ & $(B)$ & $(1 B)$ & Co-crystal formed & Co-crystal formed \\
$(1)$ & $(C)$ & $(1 C)$ & Co-crystal formed & Co-crystal formed \\
$(2)$ & $(A)$ & $(2 A)$ & Co-crystal formed & Co-crystal formed \\
$(2)$ & $(B)$ & $(2 B)$ & Co-crystal formed & Co-crystal formed \\
$(2)$ & $(C)$ & $(2 C)$ & - & Co-crystal formed \\
$(3)$ & $(A)$ & $(3 A)$ & Co-crystal formed & Co-crystal formed \\
$(3)$ & $(B)$ & $(3 B)$ & Co-crystal formed & Co-crystal formed \\
$(3)$ & $(C)$ & $(3 C)$ & - & Co-crystal formed \\
$(4)$ & $(A)$ & $(4 A)$ & - & Co-crystal formed \\
$(4)$ & $(B)$ & $(4 B)$ & Co-crystal formed & - \\
$(4)$ & $(C)$ & $(4 C)$ & - & - \\
\hline
\end{tabular}

Table 3

Summary of the hydrogen-bonding parameters $\left(\AA,^{\circ}\right)$ in the co-crystals.

\begin{tabular}{|c|c|c|c|c|c|}
\hline & $D-\mathrm{H} \cdots A$ & $D-\mathrm{H}$ & $\mathrm{H} \cdots A$ & $D \cdots A$ & $\angle D-\mathrm{H} \cdots A$ \\
\hline$(1 A)$ & $\mathrm{O} 1-\mathrm{H} 1 A \cdots \mathrm{N} 1$ & $0.96(2)$ & $1.68(2)$ & $2.635(1)$ & $179(2)$ \\
\hline \multirow[t]{6}{*}{$(1 B)$} & $\mathrm{O} 1-\mathrm{H} 1 \cdots \mathrm{N} 1$ & $0.97(2)$ & $1.67(2)$ & $2.642(1)$ & $176(2)$ \\
\hline & $\mathrm{O} 4-\mathrm{H} 4 \cdots \mathrm{N} 3$ & $0.98(2)$ & $1.65(2)$ & $2.633(1)$ & $176(2)$ \\
\hline & $\mathrm{N} 2-\mathrm{H} 2 A \cdots \mathrm{O} 6$ & $0.90(2)$ & $2.02(2)$ & $2.911(1)$ & $176(2)$ \\
\hline & $\mathrm{N} 4-\mathrm{H} 4 B \cdots \mathrm{O} 3$ & $0.89(2)$ & $2.01(2)$ & $2.904(1)$ & $179(2)$ \\
\hline & $\mathrm{N} 2-\mathrm{H} 2 B \cdots \mathrm{O} 5^{\mathrm{i}}$ & $0.91(2)$ & $2.01(2)$ & $2.908(1)$ & $171(2)$ \\
\hline & $\mathrm{N} 4-\mathrm{H} 4 C \cdots \mathrm{O} 2^{\mathrm{ii}}$ & $0.87(2)$ & $2.04(2)$ & $2.906(1)$ & $171(2)$ \\
\hline \multirow[t]{3}{*}{$(1 C)$} & $\mathrm{O} 1-\mathrm{H} 1 \cdots \mathrm{N} 1$ & $1.00(3)$ & $1.65(3)$ & $2.643(2)$ & $173(2)$ \\
\hline & $\mathrm{N} 2-\mathrm{H} 2 A \cdots \mathrm{O} 2^{\mathrm{iii}}$ & $0.94(2)$ & $1.99(2)$ & $2.921(2)$ & $173(2)$ \\
\hline & $\mathrm{N} 2-\mathrm{H} 2 B \cdots \mathrm{O} 3^{\text {iv }}$ & $0.91(2)$ & $1.97(2)$ & $2.875(2)$ & $176(2)$ \\
\hline \multirow[t]{5}{*}{$(2 A)$} & $\mathrm{O} 1-\mathrm{H} 1 \cdots \mathrm{N} 2$ & 0.84 & 1.82 & $2.63(2)$ & 161 \\
\hline & $\mathrm{O} 1 A-\mathrm{H} 1 A A \cdots \mathrm{N} 2$ & 0.84 & 1.98 & $2.79(1)$ & 164 \\
\hline & $\mathrm{O} 4-\mathrm{H} 4 \cdots \mathrm{N} 4$ & $0.98(3)$ & $1.69(3)$ & $2.652(2)$ & $168(2)$ \\
\hline & $\mathrm{O} 6-\mathrm{H} 6 \cdots \mathrm{N} 3$ & $1.00(1)$ & $1.66(1)$ & $2.662(2)$ & $176(3)$ \\
\hline & $\mathrm{O} 8-\mathrm{H} 8 A \cdots \mathrm{N} 1$ & $0.94(3)$ & $1.70(3)$ & $2.635(2)$ & $173(2)$ \\
\hline \multirow[t]{3}{*}{$(2 B)$} & $\mathrm{O} 2-\mathrm{H} 2 \cdots \mathrm{N} 1$ & $0.97(2)$ & $1.66(2)$ & $2.618(1)$ & $167(2)$ \\
\hline & $\mathrm{N} 2-\mathrm{H} 2 B \cdots \mathrm{O} 1^{\mathrm{v}}$ & $0.90(2)$ & $2.05(2)$ & $2.939(1)$ & $170(1)$ \\
\hline & $\mathrm{N} 2-\mathrm{H} 2 C \cdots \mathrm{O}^{\mathrm{vi}}$ & $0.92(2)$ & $1.96(2)$ & $2.880(1)$ & $174(1)$ \\
\hline$(3 A)$ & $\mathrm{O} 1-\mathrm{H} 1 \cdots \mathrm{N} 1$ & $0.95(2)$ & $1.67(2)$ & $2.615(1)$ & $172(2)$ \\
\hline \multirow[t]{6}{*}{$(3 B)$} & $\mathrm{O} 1-\mathrm{H} 1 \cdots \mathrm{N} 3$ & $1.00(2)$ & $1.66(2)$ & $2.665(2)$ & $175(3)$ \\
\hline & $\mathrm{O} 5-\mathrm{H} 5 A \cdots \mathrm{N} 1$ & $1.00(2)$ & $1.68(2)$ & $2.675(2)$ & $173(2)$ \\
\hline & $\mathrm{N} 2-\mathrm{H} 2 A \cdots \mathrm{O} 4^{\mathrm{vii}}$ & $0.96(3)$ & $1.96(3)$ & $2.917(2)$ & $173(2)$ \\
\hline & $\mathrm{N} 4-\mathrm{H} 4 A \cdots \mathrm{O} 8^{\text {viii }}$ & $0.96(3)$ & $1.93(3)$ & $2.878(2)$ & $175(2)$ \\
\hline & $\mathrm{N} 2-\mathrm{H} 2 B \cdots \mathrm{O} 6^{\mathrm{x}}$ & $0.90(3)$ & $2.04(3)$ & $2.934(2)$ & $173(2)$ \\
\hline & $\mathrm{N} 4-\mathrm{H} 4 B \cdots \mathrm{O} 2^{\mathrm{ix}}$ & $0.92(3)$ & $2.04(3)$ & $2.953(2)$ & $171(2)$ \\
\hline \multirow[t]{2}{*}{$(4 B)$} & $\mathrm{O} 1-\mathrm{H} 1 \cdots \mathrm{N} 1$ & $1.000(1)$ & $1.609(3)$ & 2.607 (2) & $176(3)$ \\
\hline & $\mathrm{N} 2-\mathrm{H} 2 B \cdots \mathrm{O} 5^{\mathrm{x}}$ & $0.93(2)$ & $1.94(3)$ & $2.869(3)$ & $176(2)$ \\
\hline
\end{tabular}

Symmetry codes: (i) $1+x,-1+y,+z$; (ii) $-1+x, 1+y,+z$; (iii) $1-x, \frac{1}{2}+y, \frac{1}{2}-z$; (iv) $1-x, 1-y,-z$; (v) $+x, 1+y,+z$; (vi) $-1-x,-1-y, 1-z$; (vii) $-x,-y, 2-z$; (viii) $1-x,-y, 2-z$; (ix) $2-x, 1-y, 2-z$; (x) $1-x, 1-y,-1-z$.
$\mathrm{C}-\mathrm{H}$ hydrogen atoms were located geometrically and refined using a riding model. In the case of $(2 A)$ one of the four independent molecules of 4-methylcinnamic acid was disordered, the occupancies of the fragments were determined by refining them against a free variable with the sum of the two sites set to equal 1 , the occupancies were then fixed at the refined values, restraints were applied to bond lengths and thermal displacement parameters to maintain sensible geometry in the disordered fragments. Crystal structure and refinement data are given in Table 1.

\subsection{Powder X-ray diffraction}

Powder X-ray diffraction measurements were carried out on a Bruker D8 Advance powder X-ray diffractometer fitted with a LynxEye detector using $\mathrm{Cu}$ radiation $(\lambda=1.5406 \AA)$. Data collections were made at room temperature, $2 \theta 5-50^{\circ}$, with a step size of $0.024^{\circ}$. Powder X-ray diffraction measurements were taken from each of the cinnamic acids (1)-(4), the co-crystallizers $(A)-(C)$ and all of the samples from the solvent drop grinding.

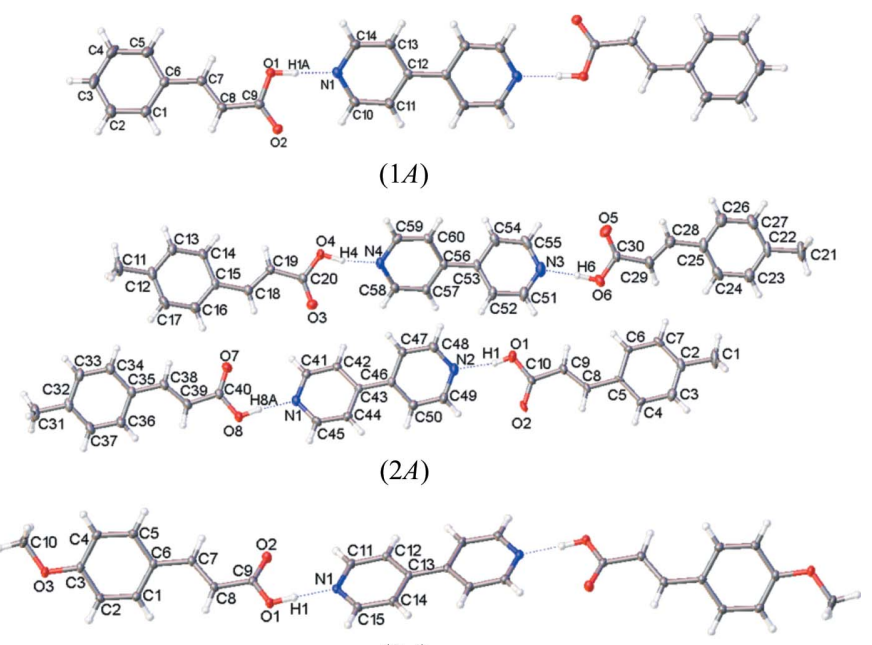

Figure 2

(3A)

Structure of $(1 A),(2 A)$ and $(3 A)$ with ellipsoids depicted at the $50 \%$ level. Hydrogen bonding is illustrated and the atom labelling shown. In structure $(2 A)$ only one position of the disordered 4-methylcinnamic acid $(\mathrm{C} 1-\mathrm{C} 10, \mathrm{O} 1, \mathrm{O} 2)$ is shown. 
Table 4

Summary of the $\pi-\pi$ contacts in the co-crystals.

\begin{tabular}{|c|c|c|c|c|}
\hline & Plane 1 & Plane 2 & $\begin{array}{l}\text { Centroid-centroid } \\
\text { distance }(\AA)\end{array}$ & $\begin{array}{l}\text { Shift } \\
\text { distance }(\AA)\end{array}$ \\
\hline$(1 A)$ & (N1, C10-C14) & $(\mathrm{N} 1, \mathrm{C} 10-\mathrm{C} 14)^{\mathrm{i}}$ & $3.951(1)$ & $1.970(2)$ \\
\hline \multirow[t]{3}{*}{$(1 B)$} & (C1-C6) & $(\mathrm{N} 3, \mathrm{C} 25-\mathrm{C} 29)^{\mathrm{i}}$ & $3.652(1)$ & $0.972(2)$ \\
\hline & (C16-C21) & $(\mathrm{N} 3, \mathrm{C} 25-\mathrm{C} 29)^{\mathrm{ii}}$ & 3.700 (1) & $0.947(2)$ \\
\hline & (N1, C10-C14) & $(\mathrm{N} 1, \mathrm{C} 10-\mathrm{C} 14)^{\mathrm{i}}$ & $3.868(1)$ & $1.822(2)$ \\
\hline$(1 C)$ & (C1-C6) & $(\mathrm{N} 1, \mathrm{C} 10-\mathrm{C} 14)^{\mathrm{iii}}$ & $3.843(1)$ & $1.557(3)$ \\
\hline \multirow[t]{6}{*}{$(2 A)$} & $(\mathrm{C} 12-\mathrm{C} 17)$ & $(\mathrm{N} 3, \mathrm{C} 51-\mathrm{C} 55)^{\mathrm{vi}}$ & $3.674(1)$ & $1.010(2)$ \\
\hline & $(\mathrm{N} 3, \mathrm{C} 51-\mathrm{C} 55)$ & $(\mathrm{N} 3, \mathrm{C} 51-\mathrm{C} 55)^{\mathrm{v}}$ & $3.703(1)$ & $1.411(3)$ \\
\hline & (N1, C41-C45) & $(\mathrm{N} 1, \mathrm{C} 41-\mathrm{C} 45)^{\mathrm{vi}}$ & $3.768(1)$ & $1.331(2)$ \\
\hline & (N1, C41-C45) & $(\mathrm{C} 2-\mathrm{C} 7)^{\mathrm{vii}}$ & $3.683(1)$ & $1.668(2)$ \\
\hline & (N1, C41-C45) & $(\mathrm{C} 2 A-\mathrm{C} 7 A)^{\mathrm{vii}}$ & 3.652 (1) & $1.451(2)$ \\
\hline & (C22-C27) & $(\mathrm{C} 22-\mathrm{C} 27)^{\mathrm{viii}}$ & $3.865(1)$ & $1.615(3)$ \\
\hline \multirow[t]{3}{*}{$(2 B)$} & (C1-C6) & $(\mathrm{C} 1-\mathrm{C} 6)^{\mathrm{ix}}$ & 3.984 (1) & $1.701(2)$ \\
\hline & (C1-C6) & $(\mathrm{N} 1, \mathrm{C} 11-\mathrm{C} 15)^{\mathrm{x}}$ & $3.825(1)$ & $1.332(2)$ \\
\hline & (N1, C11-C15) & $(\mathrm{N} 1, \mathrm{C} 11-\mathrm{C} 15)^{\mathrm{xi}}$ & $3.674(1)$ & $1.592(2)$ \\
\hline$(3 A)$ & (N1, C11-C15) & $(\mathrm{N} 1, \mathrm{C} 11-\mathrm{C} 15)^{\mathrm{xii}}$ & $3.754(1)$ & $1.825(2)$ \\
\hline$(4 B)$ & (C1-C6) & $(\mathrm{N} 1, \mathrm{C} 12-\mathrm{C} 16)^{\mathrm{xiii}}$ & 3.900 & 1.940 \\
\hline
\end{tabular}

Symmetry codes: (i) $2-x,-y, 1-z$; (ii) $-x, 2-y,-z$; (iii) $x, \frac{3}{2}-y, \frac{1}{2}+z$; (iv) $1-x, 1-y,-z$; (v) $1-x,-y,-z$; (vi) $2-x, 1-y,-z$; (vii) $2-x,-y,-z$; (viii) $x,-1+y,-1+z$; (ix) $1-x, 1-y, 2-z$; (x) $1+x, 1+y,+z$; (xi) $-x,-y, 1-z$; (xii) $1-x, 1-y, 1-z ;$ (xiii) $1+x,+y, 1+z$.

\section{Results and discussion}

\subsection{Recrystallization}

Co-crystals were successfully formed from a number of the combinations which are summarized in Table 2. Co-crystals are denoted by a combination of the cinnamic acid number and the co-crystallizer letter, e.g. ( $1 A)$ represents co-crystals of trans-cinnamic acid (1) and 4,4'-dipyridyl $(A)$.

\subsection{Di-pyridyl $(A)$}

Co-crystals $(1 A)$ and $(3 A)$ were crystallized from ethanol and THF, respectively. Both structures were obtained in the monoclinic space group $P 2_{1} / n$ with 1 molecule of the cinnamic acid derivative and half a 4,4-dipyridyl molecule in the asymmetric unit. In the case of $(2 A)$ the structure was obtained from THF in the triclinic space group $P \overline{1}$ with four molecules of (2) and two molecules of $(A)$ in the asymmetric unit (Fig. 2).

In all three co-crystal structures identified containing $(A)$, each of the $\mathrm{N}$ atoms on $(A)$ forms a hydrogen bond to the $\mathrm{OH}$ group of the carboxylic acid group on the cinnamic acid derivative (Fig. 3) alongside weak $\pi-\pi$ contacts (Tables 3, 4, S1, and Fig. S1 of the supporting information). In both ( $1 A)$

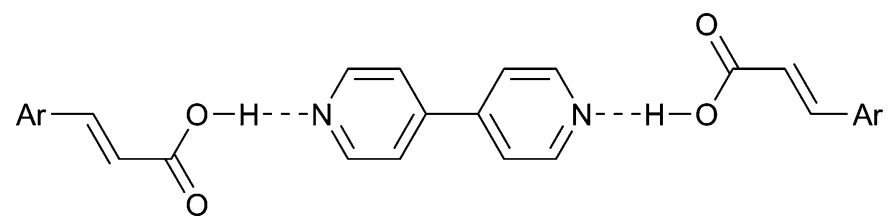

Figure 3

Illustration of the hydrogen-bonding interactions in co-crystals containing $(A)$. and $(3 A)$ the asymmetric unit contains half a molecule of $(A)$, the other half of which is generated by an inversion centre, consequently the pyridyl rings of $(A)$ are planar. In the case of $(2 A)$ the two unique molecules of $(A)$ are twisted with torsion angles of $-28.2(2)(\mathrm{C} 57-\mathrm{C} 56-\mathrm{C} 53-\mathrm{C} 52)$ and $25.5(2)^{\circ}$ (C44-C43-C46-C50) between the pyridyl rings.

Examining the fingerprint plots associated with the Hirshfeld surfaces for each of the three structures shows consistent

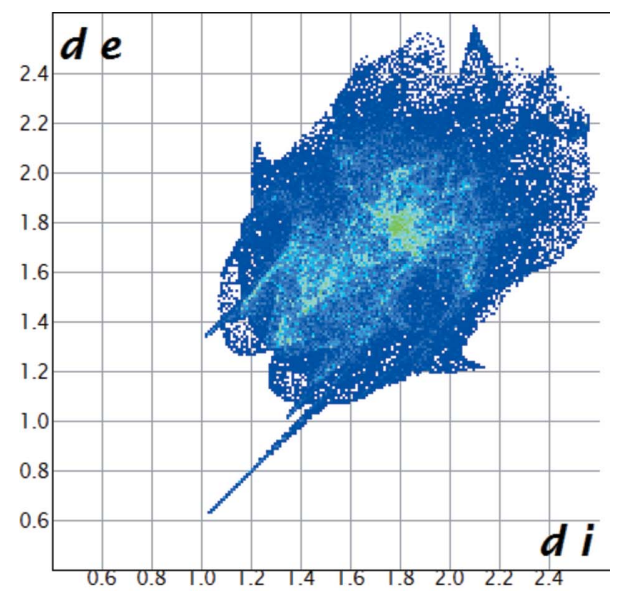

(a)

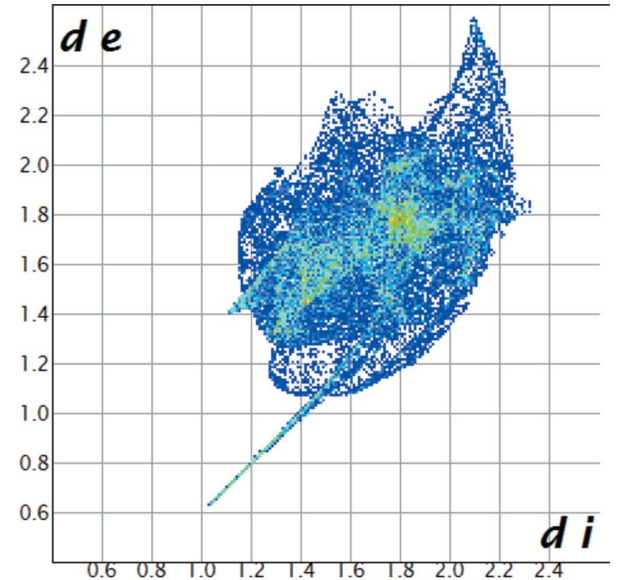

(b)

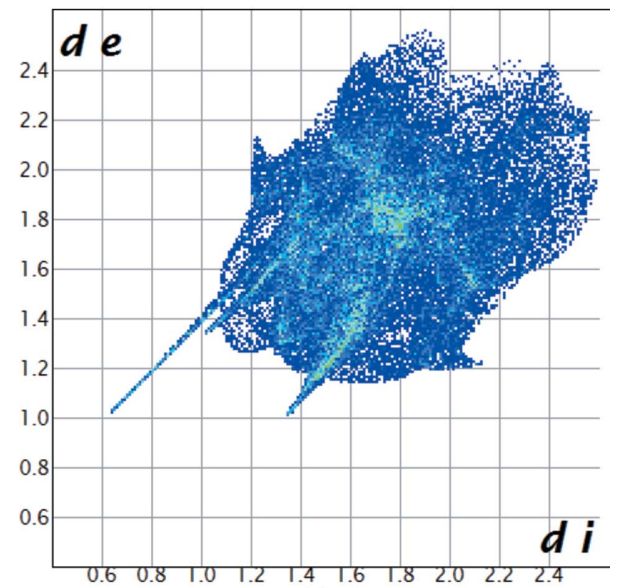

(c)

Figure 4

(1A) Fingerprint plots (a) dipyridyl and trans-cinnamic acid combined, (b) dipyridyl in the co-crystal, (c) trans-cinnamic acid in the co-crystal. 
features as would be expected from their similar structures and consequently only those for $(1 A)$ are shown in Fig. 4. The $\mathrm{O}-\mathrm{H} \cdots \mathrm{N}$ hydrogen bonding is depicted by the sharp pointed features $d e 0.6 \AA$, di $1.0 \AA$ in Figs. 4(a) and $(b)$, and $d e 1.0 \AA$, $d i$ $0.6 \AA$ in Fig. 4(c). The other feature of note is the short $\mathrm{C}-$ $\mathrm{H}$... O contacts highlighted by the short sharp spike at de $1.3 \AA$, di $1.0 \AA$ in Figs. 4(a) and (b) , and de $1.3 \AA$, di $1.0 \AA$ in Fig. 4(c).

\subsection{Iso-nicotinamide $(B)$}

Co-crystals of $(1 B)$ and $(3 B)$ were crystallized from ethanol and THF, respectively, in the triclinic space group $P \overline{1}$ with two molecules of cinnamic acid derivative [trans-cinnamic acid in $(1 B)$ and 4-methoxycinnamic acid in $(3 B)]$ and two molecules of $(B)$ in the asymmetric unit. The structure of $(1 B)$ has been previously reported (Aakeröy et al., 2002). Co-crystals of (2B)

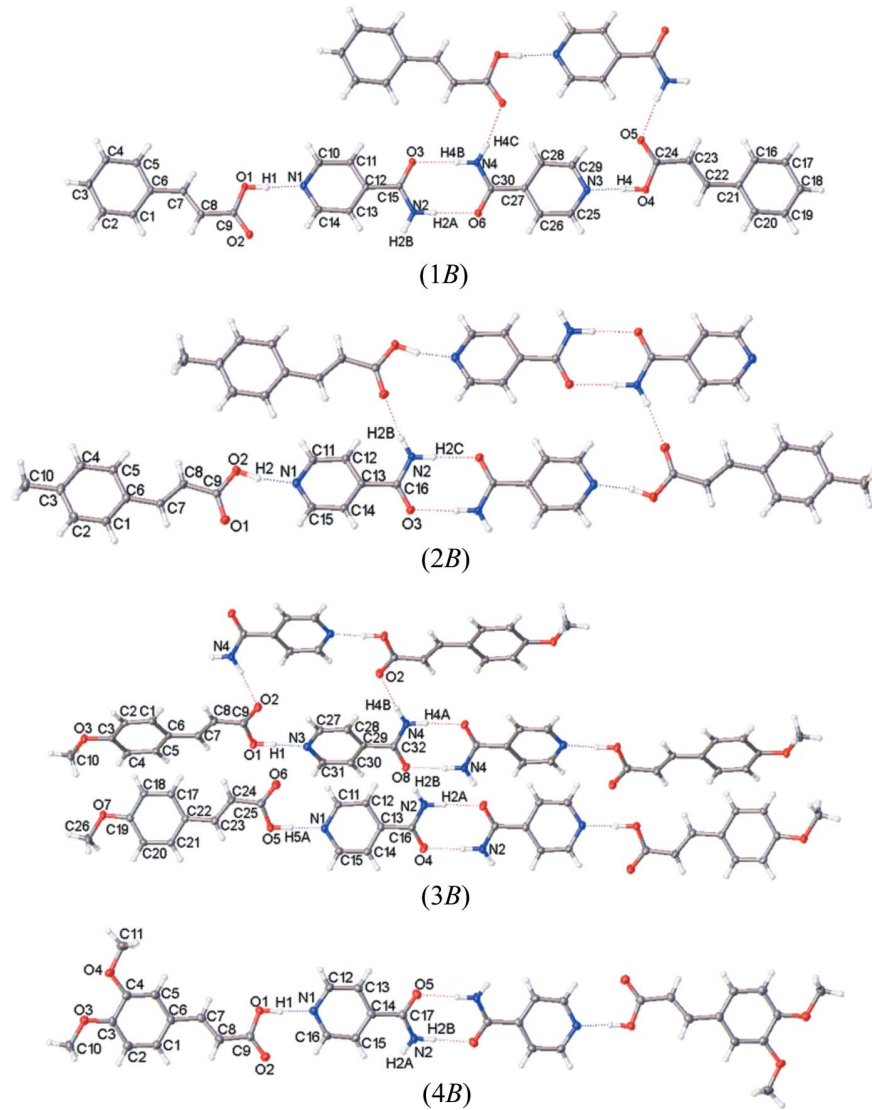

Figure 5

Structure of $(1 B),(2 B),(3 B)$ and $(4 B)$ with ellipsoids depicted at the $50 \%$ level. Hydrogen bonding is illustrated and the atom labelling shown.

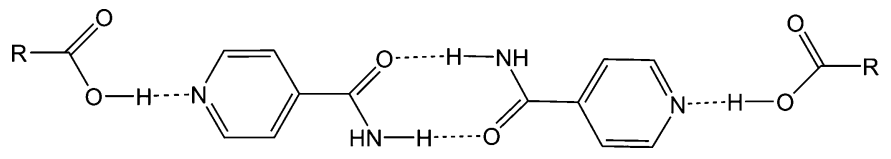

Figure 6

Illustration of the hydrogen-bonding interactions in co-crystals containing $(B)$. and $(4 B)$ crystallized from THF, in the case of $(2 B)$ in the triclinic space group $P \overline{1}$, while $(4 B)$ crystallized in the monoclinic space group $P 2_{1} / c$. Both structures contained one molecule of the cinnamic acid derivative [4-methyl cinnamic acid for $(2 B)$ and 3,4-methoxycinnamic acid for $(4 B)]$ and one molecule of $(B)$ in the asymmetric unit (Fig. 5).

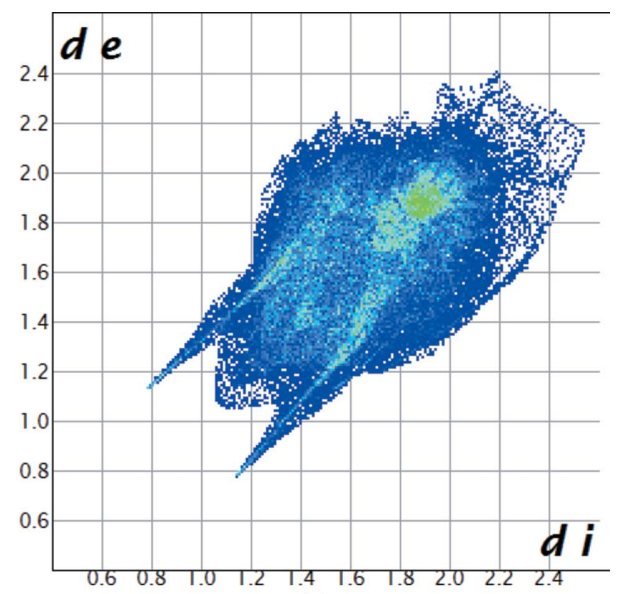

(a)

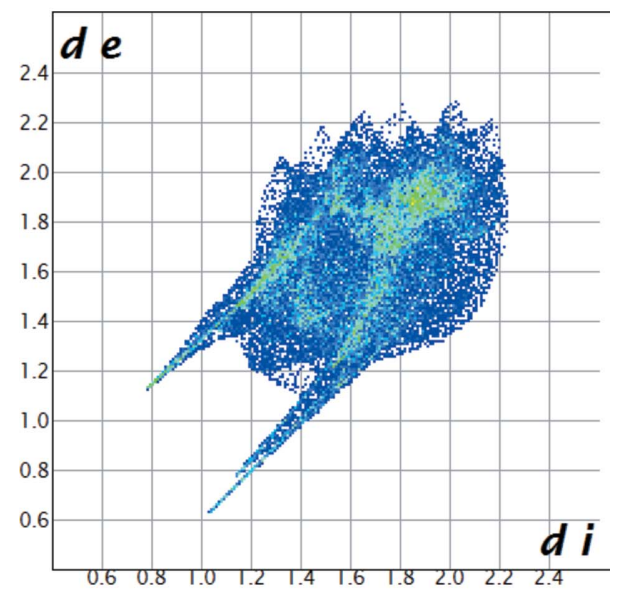

(b)

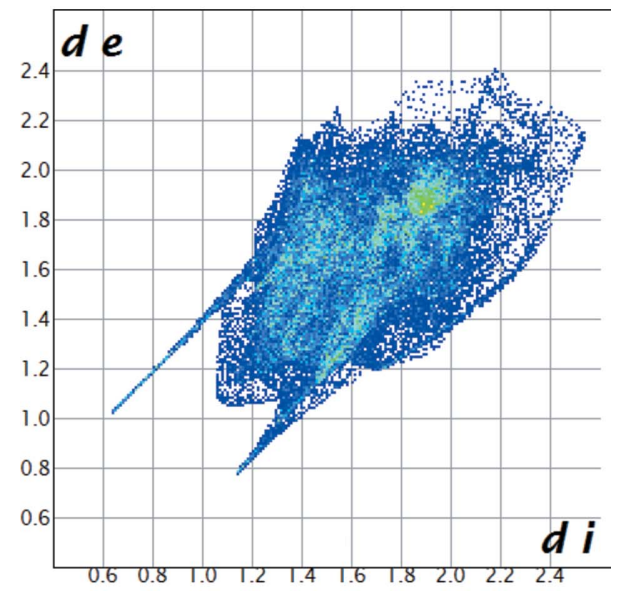

(c)

Figure 7

(1B) Fingerprint plots (a) iso-nicotinamide and trans-cinnamic acid combined, $(b)$ iso-nicotinamide in the co-crystal, $(c)$ trans-cinnamic acid in the co-crystal. 
In line with a previous study into the co-crystals of cinnamic acids and $(B)$, during which the structure of $(1 B)$ was published (Aakeröy et al., 2002), the three novel structures reported here $[(2 B),(3 B)$ and $(4 B)]$ all contain carboxylic acid $\cdots$ pyridine $(\mathrm{O}-\mathrm{H} \cdots \mathrm{N})$ and amide $\cdots$ amide hydrogenbonding interactions. This creates a hydrogen-bonded chain of four molecules terminated by cinnamic acid molecules which sandwich two molecules of $(B)$, see Fig. 6, Tables 3 and S1.

Three of the structures $[(1 B),(2 B)$ and $(3 B)]$ also displayed additional hydrogen-bonding interactions between the chains, with the $\mathrm{O}$ of the carboxylic acid and an amide $\mathrm{NH}$, creating a four molecule wide offset ribbon through the structure (Fig. $6)$. In the fourth structure, the $(4 B)$ units are twisted with respect to each other, possibly due to the bulk created by the presence of two OMe substituents. In three of the structures weak $\pi-\pi$ contacts were also identified (Table 4 , Fig. S2), the exception was in $(3 B)$.

For all four of the structures, the Hirshfeld fingerprint plots show very similar features and hence only those for $(1 B)$ are illustrated in Fig. 7. The sharp features at $d e \simeq 1.1 \AA, d i \simeq$ $0.8 \AA$ in Figs. $7(a)$ and $(b)$ and $d e \simeq 0.8 \AA, d i \simeq 1.1 \AA$ in Fig. $7(c)$, are due to the amide $\mathrm{NH} \cdots \mathrm{O}$ hydrogen-bond interactions, while the sharp features at $d e \simeq 0.8 \AA, d i \simeq 1.1 \AA$ in Figs. $7(a)$ and $(b)$, and $d e \simeq 1.1 \AA, d i \simeq 0.8 \AA$ are associated with the carboxylic acid $\mathrm{OH} \cdots \mathrm{N}$ hydrogen bonds and the amide $\mathrm{NH} \cdots \mathrm{O}$ hydrogen-bond interaction. The apron around $d e \simeq$ $1.1 \AA, d i \simeq 1.1 \AA$ are due to short $\mathrm{H} \cdots \mathrm{H}$ contacts.

\subsection{Nicotinamide $(C)$}

(1C) was crystallized from 2-propanol in the monoclinic space group $P 2_{1} / c$ with one trans-cinnamic acid molecule and one nicotinamide in the asymmetric unit, Fig. 8. The amide group of the nicotinamide is twisted out of the plane of the pyridine with a torsion angle of $-27.5(2)^{\circ}(\mathrm{C} 12-\mathrm{C} 13-\mathrm{C} 15-$ $\mathrm{N} 2)$, while a torsion angle of $-166.6(2)^{\circ}(\mathrm{C} 7-\mathrm{C} 8-\mathrm{C} 9-\mathrm{O} 1)$ is observed for the carboxylic acid. As with $(1 B)$ the structure contains hydrogen bonding between the carboxylic $\mathrm{OH}$ and the pyridine $\mathrm{N}$, amide-amide hydrogen bonding and hydrogen bonding between the $\mathrm{O}$ of the carboxylic acid group and the amide NH (Table 3 and S1). Due to the non-planar orientation of the molecules, hydrogen bonding creates a three-dimen-

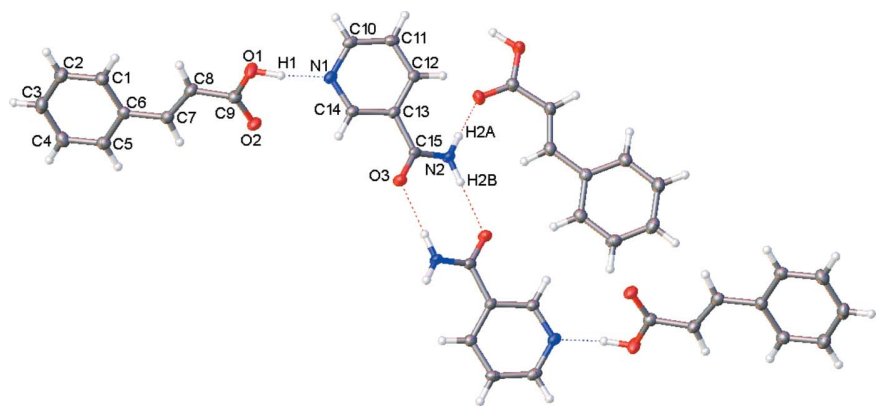

Figure 8

Structure of $(1 C)$ with ellipsoids depicted at the $50 \%$ level. Hydrogen bonding is illustrated and the atom labelling shown. sional network. In addition $\pi-\pi$ stacking contacts are observed (Table 4).

The Hirshfeld fingerprint plots for $(1 C)$ show the expected interactions. The sharp peak at $d e \simeq 0.6 \AA, d i \simeq 1.0 \AA$ in Fig. $9(b)$ and $d e \simeq 1.0 \AA, d i \simeq 0.6 \AA$ on Fig. $9(c)$ corresponds to the $\mathrm{OH} \cdots \mathrm{N}$ interactions. There is also a sharp peak at $d e \simeq 1.1 \AA$, $d i \simeq 0.8 \AA$ in Figs. $9(a)$ and $(b)$ corresponding to the $\mathrm{OH} \cdots \mathrm{N}$

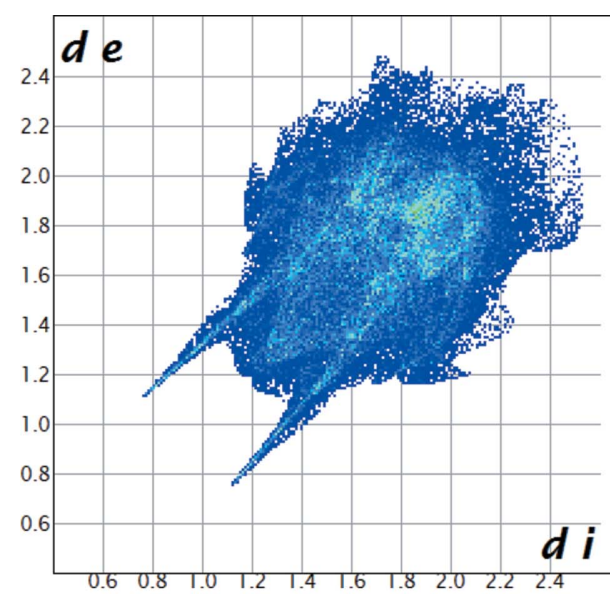

(a)

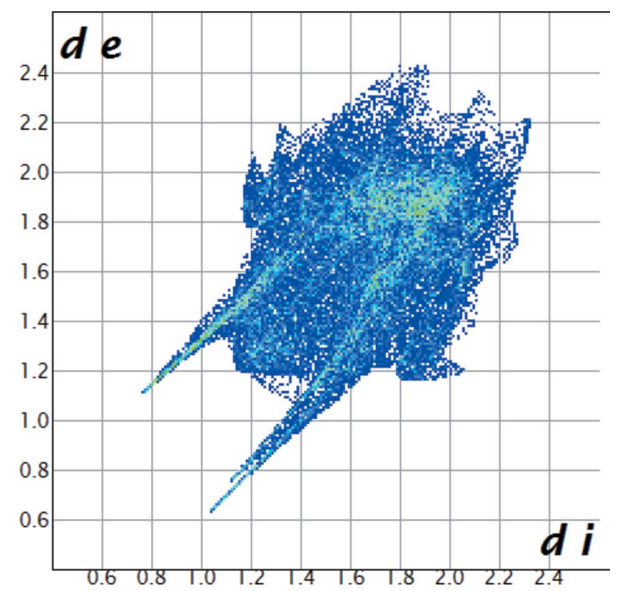

(b)

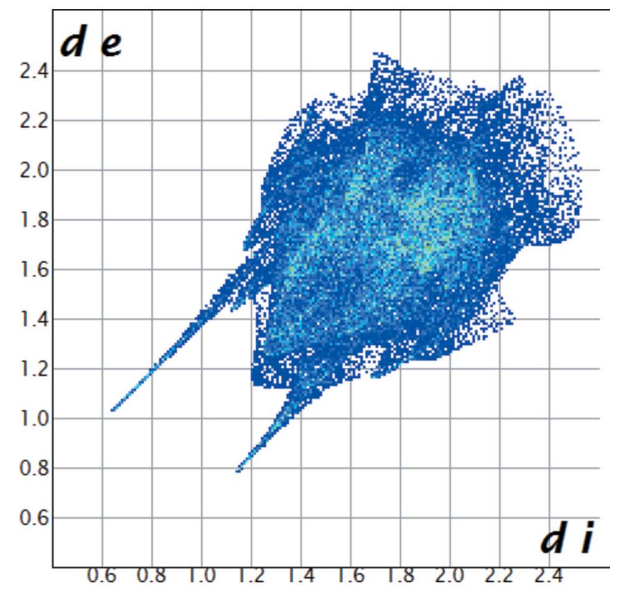

(c)

Figure 9

(1C) Fingerprint plots (a) nicotinamide and trans-cinnamic acid combined, (b) nicotinamide in the co-crystal, (c) trans-cinnamic acid in the co-crystal. 
amide-amide hydrogen bonding. The sharp peak at $d e \simeq$ $0.8 \AA, d i \simeq 1.1 \AA$ in Figs. $9(a)$ and $(c)$ relates to either the amide carboxylic $\mathrm{NH} \cdots \mathrm{O}$ or the $\mathrm{C} 10-\mathrm{H} 10 \cdots \mathrm{O} 2$ interactions.

\subsection{Solvent drop ball mill grinding}

The X-ray powder diffraction patterns of mixtures of cinnamic acid with a co-crystallizer after solvent drop grinding were compared with those of the reactants. The formation of a co-crystal was inferred by the absence of peaks corresponding to the reactants and the appearance of new peaks (see Figs. S4-S15). The presence of ethanol served to act as a catalyst in the successful formation of ten co-crystals, Table 2. None of the samples showed evidence of having formed a co-crystal following ball mill grinding under the same conditions in the absence of a small amount of ethanol. The only combinations which did not form co-crystals under the conditions employed here were those of 3,4-methoxycinnamic acid (4) with isonicotinamide $(B)$ or nicotinamide $(C)$. Powder patterns were simulated using PowderCell, Version 2.4 (Kraus \& Nolze, 1996) from the eight crystal structures obtained herein and compared with the experimental powder patterns from the solvent drop grinding. There was generally good agreement between the experimental and simulated patterns for $(1 A)$, $(1 B),(1 C),(2 A),(2 B),(3 A)$ and $(3 B)$ apart from small shifts in the peak position due to the crystal structures having been obtained at $100 \mathrm{~K}$ and the powder patterns recorded at room temperature. The only peaks in experimental patterns for $(4 B)$ and $(4 C)$ are attributable to their two respective starting components.

\section{Conclusions}

A number of co-crystals of cinnamic acid derivatives, transcinnamic acid (1), 4-methylcinnamic acid (2), 4-methoxy cinnamic acid (3) and 3,4-methoxy cinnamic acid (4), with cocrystallizers, dipyridyl $(A)$, iso-nicotinamide $(B)$ and nicotinamide $(C)$, were successfully synthesized by two different methods - recrystallization or solvent drop grinding. The solvent drop grinding resulted in ten co-crystals being obtained.

Single-crystal X-ray structures are reported for seven novel co-crystals of cinnamic acid derivatives with co-crystallizers. In the current study, single-crystal structures were not obtained for $(2 C),(3 C)$ and $(4 A)$, although their formation was implied by powder X-ray diffraction after the respective components were combined by solvent drop ball milling. The predicted product $(4 C)$ was not formed under any of the preparation conditions tested. Further, more extensive attempts to form co-crystals of $(4 C)$ under different crystallization conditions may yield success in the future. In the case of the co-crystal structures reported here, the structures are strongly influenced by structure of the co-crystallizer rather than the cinnamic acid. This is perhaps not a surprise given that the cinnamic acid derivatives selected have relatively similar structures. In the two sets of cinnamic acid co-crystals formed with either dipyridyl or iso-nicotinamide, there are key hydrogen-bonding motif features that are observed for the specific co-crystallizer, and distinctive Hirshfeld fingerprint plots. In the case of the dipyridyl co-crystals, hydrogen-bonding interactions are formed between the $\mathrm{OH}$ group on the carboxylic acid and the pyridyl $\mathrm{N}$, resulting in two cinnamic acid molecules per dipyridyl. The cinnamic acid derivative:iso-nicotinamide cocrystals formed in a 1:1 ratio and in-line with other cinnamic acid derivative:iso-nicotinamide co-crystal structures previously reported (Aakeröy et al., 2002): hydrogen-bonding interactions were observed between the carboxylic acid $\mathrm{OH}$ and the $\mathrm{N}$ of the pyridyl, alongside amide-amide hydrogenbonding interactions. No conclusions can be drawn about trends for cinnamic acid:nicotinamide co-crystals as only one 1:1 co-crystal $(1 C)$ was formed in the current study. The cocrystal structure of $(1 C)$ contained the same hydrogenbonding interactions as those found for the cinnamic acid:isonicotinamide co-crystals. As noted in $\$ 1$ the few examples of hydrates of cinnamic acid derivative co-crystals with isonicotinamide or nicotinamide have resulted in the formation of different hydrogen-bonding motifs to those observed here (Clarke et al., 2010; Bevill et al., 2014). In all of the co-crystal structures reported there was no evidence to suggest that proton transfer occurred within the hydrogen bonds to form a salt, based on the fact that the $X-D$ bond lengths were consistent with expected values and $\mathrm{H}$ atoms were located in the difference map within expected $D-\mathrm{H}$ bonding distances.

\section{Acknowledgements}

The authors would like to thank Jason Potticary for his assistance setting up the ball mill.

\section{References}

Aakeröy, C. B., Chopade, P. D., Ganser, C., Rajbanshi, A. \& Desper, J. (2012). CrystEngComm, 14, 5845-5853.

Aakeröy, C. B. (1997). Acta Cryst. B53, 569-586.

Aakeröy, C. B., Beatty, A. M. \& Helfrich, B. A. (2002). J. Am. Chem. Soc. 124, 14425-14432.

Aakeröy, C. B., Fasulo, M. E. \& Desper, J. (2007). Mol. Pharm. 4, $317-$ 322.

Aitipamula, S. et al. (2012). Cryst. Growth Des. 12, 2147-2152.

Allen, F. H., Mahon, M. F., Raithby, P. R., Shields, G. P. \& Sparkes, H. A. (2005). New J. Chem. 29, 182-187.

Allen, F. H., Motherwell, W. D. S., Raithby, P. R., Shields, G. P. \& Taylor, R. (1999). New J. Chem. 23, 25-34.

Allen, F. H., Shields, G. P., Taylor, R., Allen, F. H., Raithby, P. R., Shields, G. P. \& Taylor, R. (1998). Chem. Commun. pp. 1043-1044.

Bevill, M. J., Vlahova, P. I. \& Smit, J. P. (2014). Cryst. Growth Des. 14, 1438-1448.

Braga, D., Maini, L. \& Grepioni, F. (2013). Chem. Soc. Rev. 42, 76387648.

Brittain, H. G. (2012). Cryst. Growth Des. 12, 5823-5832.

Bruker (2001). SADABS, Version 2012.1. Bruker AXS Inc., Madison, Wisconsin, USA.

Bučar, D.-K., Henry, R. F., Zhang, G. G. Z. \& MacGillivray, L. R. (2014). Cryst. Growth Des. 14, 5318-5328.

Clarke, H. D., Arora, K. K., Bass, H., Kavuru, P., Ong, T. T., Pujari, T., Wojtas, L. \& Zaworotko, M. J. (2010). Cryst. Growth Des. 10, 2152 2167.

Dolomanov, O. V., Bourhis, L. J., Gildea, R. J., Howard, J. A. K. \& Puschmann, H. (2009). J. Appl. Cryst. 42, 339-341. 
Elder, D. P., Holm, R. \& de Diego, H. L. (2013). Int. J. Pharm. 453, 88100.

Hemamalini, M., Loh, W.-S., Quah, C. K. \& Fun, H.-K. (2014). Chem. Cent. J. 8, 31 .

Howard, J. A. K., Mahon, M. F., Raithby, P. R. \& Sparkes, H. A. (2009). Acta Cryst. B65, 230-237.

James, S. L. et al. (2012). Chem. Soc. Rev. 41, 413-447.

Jones, J. T. A., Hasell, T., Wu, X., Bacsa, J., Jelfs, K. E., Schmidtmann, M., Chong, S. Y., Adams, D. J., Trewin, A., Schiffman, F., Cora, F., Slater, B., Steiner, A., Day, G. M. \& Cooper, A. I. (2011). Nature, 474, 367-371.

Kraus, W. \& Nolze, G. (1996). J. Appl. Cryst. 29, 301-303.

Mahon, M. F., Raithby, P. R. \& Sparkes, H. A. (2008). CrystEngComm, 10, 573-576.
Moragues-Bartolome, A. M., Jones, W. \& Cruz-Cabeza, A. J. (2012). CrystEngComm, 14, 2552-2559.

Palatinus, L. \& Chapuis, G. (2007). J. Appl. Cryst. 40, 786-790.

Palatinus, L., Prathapa, S. J. \& van Smaalen, S. (2012). J. Appl. Cryst. 45, 575-580.

Palatinus, L. \& van der Lee, A. (2008). J. Appl. Cryst. 41, 975-984.

Seaton, C. C. (2011). CrystEngComm, 13, 6583-6592.

Sheldrick, G. M. (2015). Acta Cryst. C71, 3-8.

Steed, J. W. (2013). Trends Pharmacol. Sci. 34, 185-193.

Yates, J. L. R. \& Sparkes, H. A. (2013). CrystEngComm, 15, 35473553.

Zhu, W., Yi, Y., Zhen, Y. \& Hu, W. (2015). Small, 11, 2150-2156.

Zhu, W., Zhen, Y., Dong, H., Fu, H. \& Hu, W. (2014). Prog. Chem. 26 , 1292-1306. 